Research article

Open Access

\title{
Glucosamine prevents in vitro collagen degradation in chondrocytes by inhibiting advanced lipoxidation reactions and protein oxidation
}

\author{
Moti L Tiku, Haritha Narla, Mohit Jain and Praveen Yalamanchili
}

\author{
Department of Medicine, University of Medicine and Dentistry of New Jersey, Robert Wood Johnson Medical School, One Robert Wood Johnson \\ Place, New Brunswick, NJ 08903, USA \\ Corresponding author: Moti L Tiku, tikuml@umdnj.edu
}

Received: 2 Nov 2006 Revisions requested: 10 Jan 2007 Revisions received: 5 Jul 2007 Accepted: 8 Aug 2007 Published: 8 Aug 2007

Arthritis Research \& Therapy 2007, 9:R76 (doi:10.1186/ar2274)

This article is online at: http://arthritis-research.com/content/9/4/R76

(C) 2007 Tiku et al.; licensee BioMed Central Ltd.

This is an open access article distributed under the terms of the Creative Commons Attribution License (http://creativecommons.org/licenses/by/2.0), which permits unrestricted use, distribution, and reproduction in any medium, provided the original work is properly cited.

\begin{abstract}
Osteoarthritis (OA) affects a large segment of the aging population and is a major cause of pain and disability. At present, there is no specific treatment available to prevent or retard the cartilage destruction that occurs in OA. Recently, glucosamine sulfate has received attention as a putative agent that may retard cartilage degradation in $O A$. The precise mechanism of action of glucosamine is not known. We investigated the effect of glucosamine in an in vitro model of cartilage collagen degradation in which collagen degradation induced by activated chondrocytes is mediated by lipid peroxidation reaction. Lipid peroxidation in chondrocytes was measured by conjugated diene formation. Protein oxidation and aldehydic adduct formation were studied by immunoblot assays. Antioxidant effect of glucosamine was also tested on malondialdehyde (thiobarbituric acid-reactive substances [TBARS]) formation on purified lipoprotein oxidation for comparison. Glucosamine sulfate and glucosamine hydrochloride in millimolar $(0.1$ to 50$)$ concentrations specifically and significantly inhibited collagen degradation
\end{abstract}

induced by calcium ionophore-activated chondrocytes. Glucosamine hydrochloride did not inhibit lipid peroxidation reaction in either activated chondrocytes or in copper-induced oxidation of purified lipoproteins as measured by conjugated diene formation. Glucosamine hydrochloride, in a dosedependent manner, inhibited malondialdehyde (TBARS) formation by oxidized lipoproteins. Moreover, we show that glucosamine hydrochloride prevents lipoprotein protein oxidation and inhibits malondialdehyde adduct formation in chondrocyte cell matrix, suggesting that it inhibits advanced lipoxidation reactions. Together, the data suggest that the mechanism of decreasing collagen degradation in this in vitro model system by glucosamine may be mediated by the inhibition of advanced lipoxidation reaction, preventing the oxidation and loss of collagen matrix from labeled chondrocyte matrix. Further studies are needed to relate these in vitro findings to the retardation of cartilage degradation reported in $\mathrm{OA}$ trials investigating glucosamine.

\section{Introduction}

Osteoarthritis $(O A)$ is characterized by the progressive degradation and loss of articular cartilage [1]. OA is the most common arthritic disease and its incidence increases with age. As population demographics changes to include more elderly individuals, this disease will have a serious impact in multiple ways. Along with the cost for health care and lost work time, individuals with OA suffer from pain and disability [2]. Currently, there is no specific treatment to prevent or retard the cartilage degradation in OA. Present treatments used for $\mathrm{OA}$ provide only symptomatic relief from the pain. Glucosamine sulfate, which has received attention as a putative agent that may retard cartilage structural degradation in OA, has been investigated in several OA trials [3-5]. The result on applicability of glucosamine in the clinical setting is still controversial [68]. Glucosamine in its various salt formulations with or without chondroitin sulfate is available over-the-counter as a nutritional

AGE = advanced glycation reaction; BSA = bovine serum albumin; $\mathrm{Cu}=$ copper; DMEM = Dulbecco's modified Eagle's medium; DNP = dinitrophenyl; EBSS = Earl's balanced salt solution; $E C L=$ enhanced chemiluminescence; $F B S=$ fetal bovine serum; HBSS = Hanks' balanced salt solution; $\mathrm{HRP}=$ horseradish peroxidase; IL-1 = interleukin-1; LDL = low-density lipoprotein; OA = osteoarthritis; PBS = phosphate-buffered saline; TBARS = thiobarbituric acid-reactive substances; TBS $=$ Tris-buffered saline. 
supplement and is consumed by large numbers of osteoarthritic patients.

The mechanism of retardation of cartilage degradation by glucosamine is not known. Glucosamine has been shown to have a number of effects in in vitro chondrocyte and explant cultures [9-13]. These effects include stimulation of proteoglycan synthesis, inhibition of the degradation of proteoglycans, and inhibition of matrix metalloproteinase-3 synthesis [14-16]. Glucosamine inhibits aggrecanase activity via suppression of glycosylphosphatidylinositol-linked proteins [17]. Furthermore, glucosamine has been shown to inhibit cytokine (interleukin-1 [IL-1])-induced activation of chondrocytes and nuclear factorkappa-B activity and to upregulate type II IL-I decoy receptor $[18,19]$. In vivo, glucosamine helps enhance healing of cartilage injury [20-23]. Glucosamine has been demonstrated to have immunosuppressive and tumor-inhibiting activity $[24,25]$. All these pleiotropic effects of glucosamine may individually or collectively have a chondroprotective effect.

Does the ability of glucosamine sulfate to retard cartilage structural degradation observed in OA clinical studies [3-5] involve the protection of collagen degradation? We tested the effect of glucosamine in an in vitro model of chondrocytedependent collagen degradation [26] in which collagen degradation is mediated mostly by the activation of chondrocyte lipid peroxidation resulting in aldehydic oxidation and fragmentation of cartilage collagen.

\section{Materials and methods Reagents}

Calcium ionophore A23187, vitamin E, butylated hydroxytoluene, tetramethoxypropane, glucose oxidase, glucosamine hydrochloride (interchangeably described as glucosamine), and other reagents were purchased from Sigma-Aldrich (St. Louis, MO, USA). Rotta Research Laboratorium (Monza, Italy) provided glucosamine sulfate. Hydrogen peroxide of reagent grade was obtained from Fisher Scientific (part of Thermo Fisher Scientific Inc., Waltham, MA, USA). Dulbecco's modified Eagle's medium (DMEM), fetal bovine serum (FBS), Hanks' balanced salt solution (HBSS), Earl's balanced salt solution (EBSS), L-glutamine, gentamicin, HEPES buffer, penicillin, and streptomycin were purchased from Gibco-BRL (now part of Invitrogen Corporation, Carlsbad, CA, USA). Proline, $L[2,3,4,5-H]$ with specific activity of 90 curies per millimole was obtained from American Radiolabeled Chemicals, Inc. (St. Louis, MO, USA).

\section{Isolation of rabbit articular chondrocytes}

NZW rabbits (2.2 to $2.9 \mathrm{~kg}$ ) of either gender were killed by intravenous injection of Beuthanasia-D special (ScheringPlough Corporation, Kenilworth, NJ, USA). The chondrocytes were isolated as described previously [26]. The viability of chondrocytes was confirmed by trypan blue exclusion. Primary chondrocytes were suspended in 10\% FBS in DMEM contain- ing antibiotics (1\%) and HEPES buffer (10 mM, pH 7.4) (complete media).

\section{Experimental design}

Primary rabbit articular chondrocytes were distributed into 24well plates at a concentration of 1 to $2 \times 10^{5}$ cells per well in $1 \mathrm{ml}$ of complete media. Chondrocytes were allowed to attach for 3 to 5 days, and media were changed every 3 days. Confluent cells in multiwell plates were labeled with 1 to $2 \mu \mathrm{C} /$ well with $\left[{ }^{3} \mathrm{H}\right]$-proline during the last 24 to 48 hours of cell culture. The cell monolayer was washed at least four to five times with warm HBSS by flipping the plates to remove unincorporated proline from the matrix. Albumin- or serum-free EBSS was added to wells. Experiments were carried out in triplicate wells. The test reagents were added, and the total volume was adjusted to $0.5 \mathrm{ml}$ with EBSS. The cultures were incubated at $37^{\circ} \mathrm{C}$ in a humidified $5 \% \mathrm{CO}_{2}$ incubator for 4 to 24 hours. $\left[{ }^{3} \mathrm{H}\right]$-proline release was measured in cell supernatant and cell lysates. A 100- $\mu$ l aliquot was removed and processed for scintillation counting. The plastic-bound $\left[{ }^{3} \mathrm{H}\right]$-proline-labeled matrix (that is, residuum) was solubilized with $0.5 \mathrm{M} \mathrm{NaOH}$ and counted. Percentage release of total $\left[{ }^{3} \mathrm{H}\right]$-proline-labeled collagen was calculated.

\section{Lipoprotein and lipoprotein oxidation}

The very-low-density lipoprotein and low-density lipoprotein (LDL) fractions were isolated from serum by ultracentrifugation at a density of $1.063 \mathrm{~g} / \mathrm{ml}$ and were kindly provided by Vincent A. Rifici and Avedis K. Khachadurian from the Department of Medicine of our medical school [27]. Lipoproteins were tested for susceptibility for oxidation in incubation with or without glucosamine. Lipoprotein $(0.25$ to $0.5 \mathrm{mg} / \mathrm{ml})$ was incubated at $30^{\circ} \mathrm{C}$ in phosphate-buffered saline (PBS) for 4 hours in the absence or presence of $5 \mu \mathrm{M} \mathrm{Cu}^{2+}$ (copper ion) or $5 \mu \mathrm{M} \mathrm{Cu}^{2+}$ and 50,5 , or $0.5 \mathrm{mM}$ glucosamine. Data are expressed as malondialdehyde (thiobarbituric acid-reactive substances [TBARS]) equivalents in nanometers.

\section{Thiobarbituric acid-reactive substances}

Two-hundred-microliter samples of TBARS that contained 50 $\mu \mathrm{g}$ of lipoprotein proteins were assayed by incubation with 1 $\mathrm{ml}$ of $1 \%$ thiobarbituric acid for 40 minutes at $90^{\circ} \mathrm{C}$. The reaction tubes were cooled and centrifuged at $500 \mathrm{~g}$ for $10 \mathrm{~min}$ utes at $25^{\circ} \mathrm{C}$, and the absorbencies of the supernatants were measured in a spectrophotometer at $532 \mathrm{~nm}$. TBARS are expressed as nanomoles of malondialdehyde equivalents of lipoprotein protein compared with tetramethoxypropane standard [27].

\section{Conjugated diene formation}

A washed monolayer of primary articular chondrocytes in a 60$\mathrm{mm}$ Petri dish was stimulated in the presence or absence of calcium ionophore A23187 $(20 \mu \mathrm{M})$ with or without glucosamine or vitamin $E(250 \mu \mathrm{M})$ in phenol-free EBSS. The media were monitored for conjugated diene formation at 234 
$\mathrm{nm}$ at different time points [28]. Delta absorbance was expressed as absorbance at different time points minus the absorbance at 0 hour. Conjugated diene in lipoproteins was determined directly by measuring the change in absorbance at $234 \mathrm{~nm}$ of the lipoprotein samples after incubation with $\mathrm{Cu}$. Samples that contained $50 \mu \mathrm{g}$ of protein were diluted $1: 5$ with PBS before measurement, and results were expressed as difference in absorbance at $234 \mathrm{~nm}$.

\section{Preparation of cell matrix extracts}

Primary articular chondrocytes in high density $\left(1 \times 10^{6} / \mathrm{ml}\right)$ were cultured in 60-mm Petri dishes to confluence, washed three times with HBSS, and set in EBSS, with or without agonist, in a total volume of $1.5 \mathrm{ml}$ for variable durations. The medium and cell matrix were harvested with a cell scraper in the presence of a cocktail of protease inhibitors with EDTA (ethylenediaminetetraacetic acid), and the material was transferred to microcentrifuge tubes. One hundred fifty microliters of saturated trichloroacetic acid solution was added, and the tubes were incubated for 30 minutes on ice and microcentrifuged at $12,500 \mathrm{rpm}$ for 10 minutes. The supernatants were discarded, and pellets were washed with $50 \mu \mathrm{l}$ of ethanol and then suspended in $100 \mu \mathrm{l}$ of sample buffer (29) and frozen at $-70^{\circ} \mathrm{C}$. The samples were thawed and boiled for 5 minutes with $5 \mu \mathrm{l}$ of $\beta$-mercaptoethanol and later cooled on ice, vortexed, spun, and boiled as necessary. A total of $30 \mu$ of each sample was loaded onto a $4 \%$ stacking gel and separated in $10 \%$ resolving SDS-PAGE gel in a mini-PROTEAN II electrophoresis cell (Bio-Rad Laboratories, Inc., Hercules, CA, USA). Electrophoresis was carried out under the reducing condition of Laemmli [29]. Proteins were stained with Coomassie Brilliant Blue.

\section{Immunodetection of aldehyde-protein adducts}

Proteins separated by SDS-PAGE were transferred to a nitrocellulose membrane with Trans-Blot electrophoretic transfer. The blots were incubated with $50 \mathrm{ml}$ of $5 \%$ bovine serum albumin (BSA) with Tris-buffered saline (TBS) $(20 \mathrm{mM}$ Tris/500 $\mathrm{mM} \mathrm{NaCl}, \mathrm{pH} 7.5)$ containing $0.1 \%$ Tween-20 and then were washed three times for 15 minutes with $0.5 \%$ BSA with TBS. For immunodetection, blots were incubated with antibodies diluted in 1\% BSA/TBS overnight. The MDA2 mouse monoclonal antibodies, specific for malondialdehyde-modified lysine, were kindly provided by Wulf Palinski, of the University of California, San Diego (CA, USA) [30]. The monoclonal antibodies were used at dilutions of 1:2,500. The primary antibody was removed, and the blots were washed three times (15 minutes each) with TBS-containing Tween-20. The blots were then incubated in horseradish peroxidase (HRP)-labeled goat anti-mouse immunoglobulin $\mathrm{G}$ in $1 \% \mathrm{BSA} / \mathrm{TBS}$ (diluted $1: 2,500)$ for 1 hour at room temperature. Blots were again washed with TBS (15 minutes each), and proteins were visualized as outlined in the enhanced chemiluminescence (ECL) Western blotting protocol (Amersham, now part of GE Healthcare, Little Chalfont, Buckinghamshire, UK).
Figure 1

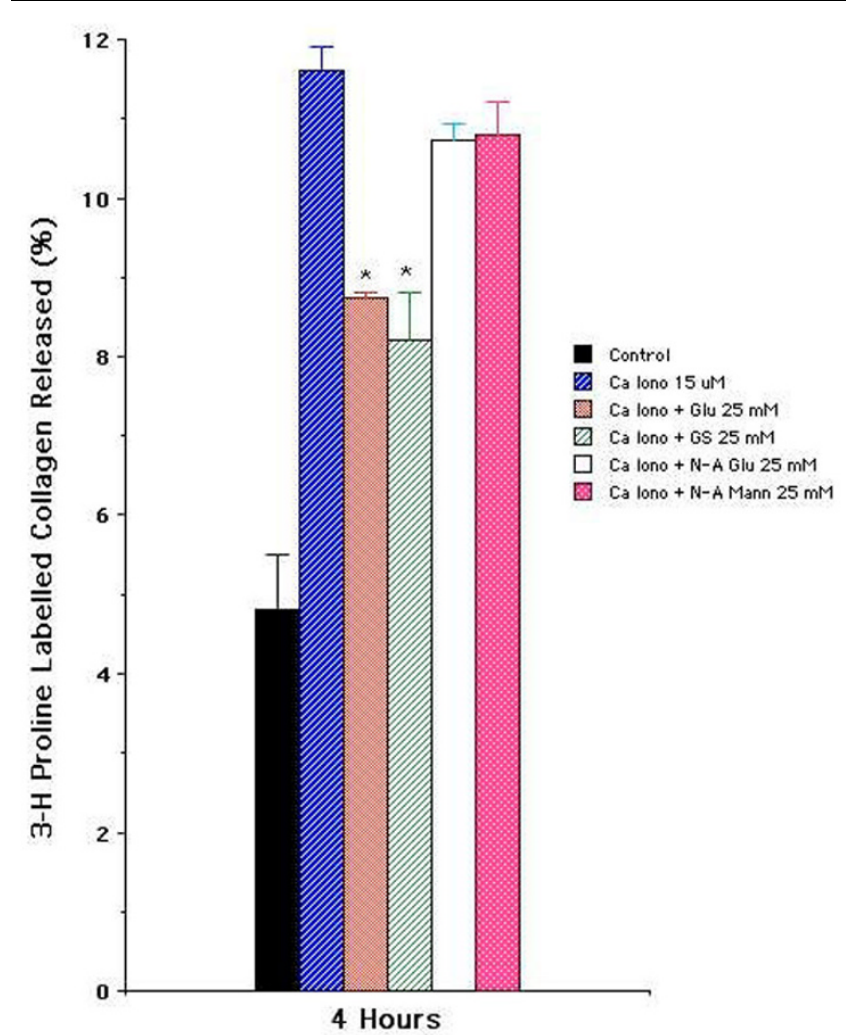

Effect of glucosamine-derived compounds on calcium ionophoreinduced release of $\left[{ }^{3} \mathrm{H}\right]$-proline-labeled articular collagen matrix. $\left[{ }^{3} \mathrm{H}\right]-$ proline-labeled monolayer of primary articular chondrocytes in 24-well plates was stimulated with calcium ionophore A23187 $(15 \mu \mathrm{M})$ in the presence or absence of glucosamine hydrochloride (Glu) $(25 \mathrm{mM})$, glucosamine sulfate (GS) $(25 \mathrm{mM}), N$-acetyl glucosamine (N-A Glu) $(25$ $\mathrm{mM})$, and $N$-acetyl mannosamine (N-A Mann) $(25 \mathrm{mM})$. The 4-hour percentage release of labeled matrix collagen is shown. The results are presented as the mean of triplicate sets of wells \pm standard error. A representative of three experiments is shown. *Statistically significant between cells stimulated with calcium ionophore and with Glu or GS. Ca lono, calcium ionophore.

\section{Immunodetection of protein-bound 2,4- dinitrophenylhydrazones}

Derivatization with dinitrophenylhydrazones was performed as published [31]. Proteins separated by SDS-PAGE were transferred as above. For immunodetection, anti-dinitrophenyl (DNP) antibody was supplied by DAKO (Dako North America, Inc., Carpinteria, CA, USA') (V401) and used at a dilution of $1: 4,000$. The secondary antibody was goat anti-rabbit antibody conjugated with HRP as outlined above in the ECL Western blotting protocol (GE Healthcare).

\section{Statistical analysis}

Results are expressed as means \pm standard error of the mean. There was a $10 \%$ coefficient of variation between the mean and highest and lowest counts in random wells of each experiment. The differences of the means between groups in the same experiment were evaluated by Student $t$ test (Statview ${ }^{\circledR}$ 

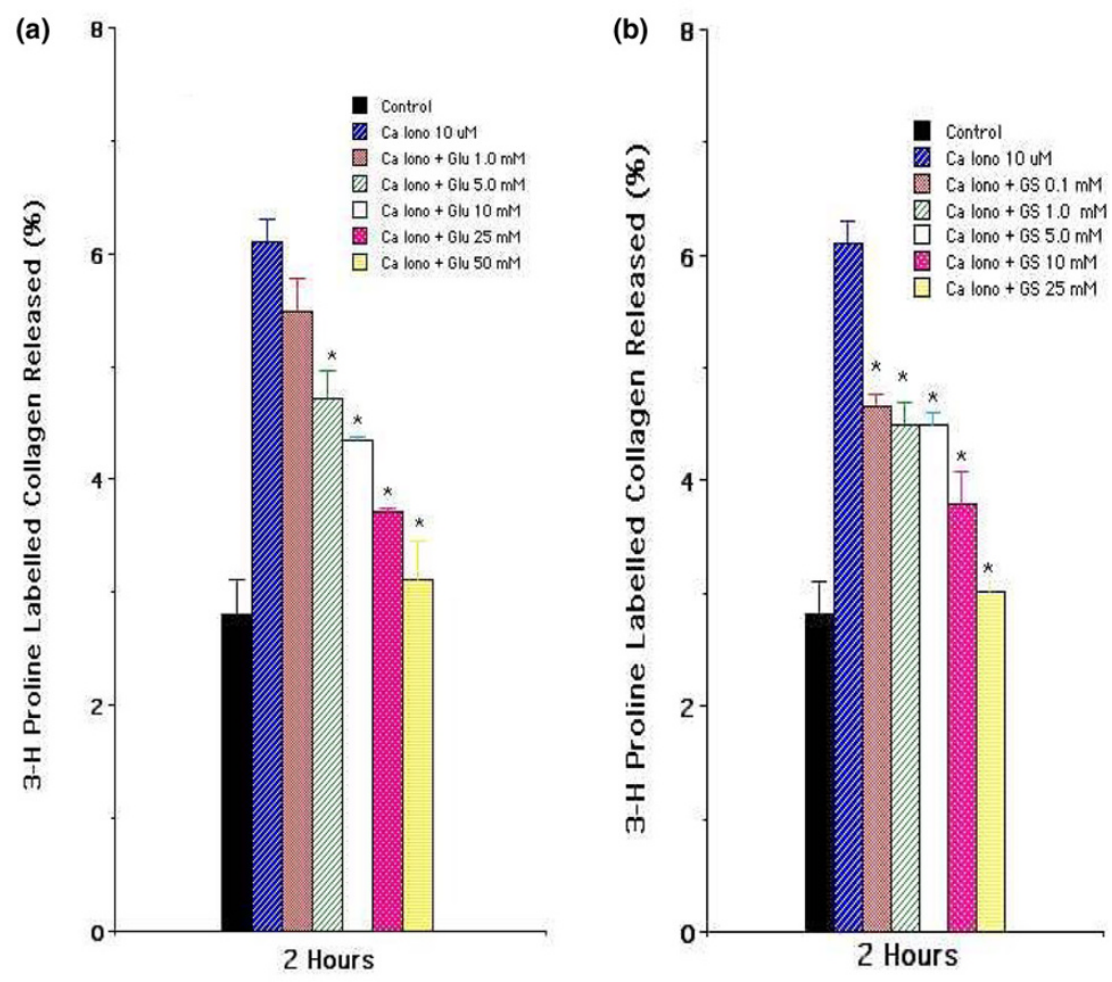

Dose-dependent effect of glucosamine hydrochloride (a) and glucosamine sulfate $(\mathbf{b})$ on release of $\left[{ }^{3} \mathrm{H}\right]$-proline-labeled collagen matrix by activated chondrocytes. $\left[{ }^{3} \mathrm{H}\right]$-proline-labeled monolayer of primary articular chondrocytes was stimulated with $\mathrm{A} 23187(10 \mu \mathrm{M})$ in the absence or presence of increasing concentrations of glucosamine hydrochloride and glucosamine sulfate. The results are presented as the mean of triplicate set of wells \pm standard error. A representative experiment is shown. *Statistically significant between cells stimulated with calcium ionophore and with glucosamine hydrochloride or glucosamine sulfate. Ca lono, calcium ionophore; Glu, glucosamine hydrochloride; GS, glucosamine sulfate.

program; SAS Institute Inc: Cary, NC USA). $P$ values less than or equal to 0.05 were considered statistically significant.

\section{Results}

\section{Glucosamine hydrochloride and glucosamine sulfate inhibit calcium ionophore-induced chondrocyte- dependent collagen degradation}

We tested the effect of glucosamine hydrochloride and glucosamine sulfate on chondrocyte-dependent collagen degradation in the previously described in vitro model [26]. For comparison and specificity, we also tested the effect of $N$ acetyl glucosamine and $N$-acetyl mannosamine. As shown in Figure 1, chondrocytes stimulated with calcium ionophore A23187 $(15 \mu \mathrm{M})$ enhanced the release of $\left[{ }^{3} \mathrm{H}\right]$-proline-labeled collagen as compared with the background amount of collagen released by unstimulated control chondrocytes. In the presence of $25 \mathrm{mM}$ concentrations of glucosamine hydrochloride or glucosamine sulfate, there was statistically significant inhibition of the release of labeled collagen at 4 hours. In comparison, $N$-acetyl glucosamine and $N$-acetyl mannosamine did not result in inhibition of collagen degradation. The data indicate that glucosamine hydrochloride and glucosamine sulfate have specificity and significantly inhibit collagen degradation by activated chondrocytes.
Dose and time effect of glucosamine hydrochloride and glucosamine sulfate on collagen degradation

As shown in Figure 2, increasing the concentration of both the glucosamine hydrochloride and glucosamine sulfate resulted in a dose-dependent inhibition of collagen degradation in calcium ionophore-stimulated chondrocyte cultures, suggesting a dose-dependent inhibitory activity on collagen degradation. Glucosamine hydrochloride (50 mM) was added at 0, 0.5, 1, 1.5 , and 2 hours after stimulation of chondrocytes by calcium ionophore $(10 \mu \mathrm{M})$ and collagen release monitored at the end of 4 hours. Addition of glucosamine hydrochloride at 0 hours resulted in significant inhibition of collagen release; a significant inhibitory effect persisted in replicate sets of cultures in which glucosamine hydrochloride was added at different time points (Figure 3). As the addition of glucosamine hydrochloride was delayed, the amount of inhibition tended to decrease but was still present. The data suggest that inhibition of collagen degradation involves downstream events of chondrocyte activation rather than interference or blockade of the early events of chondrocyte activation by calcium ionophore. 
Figure 3

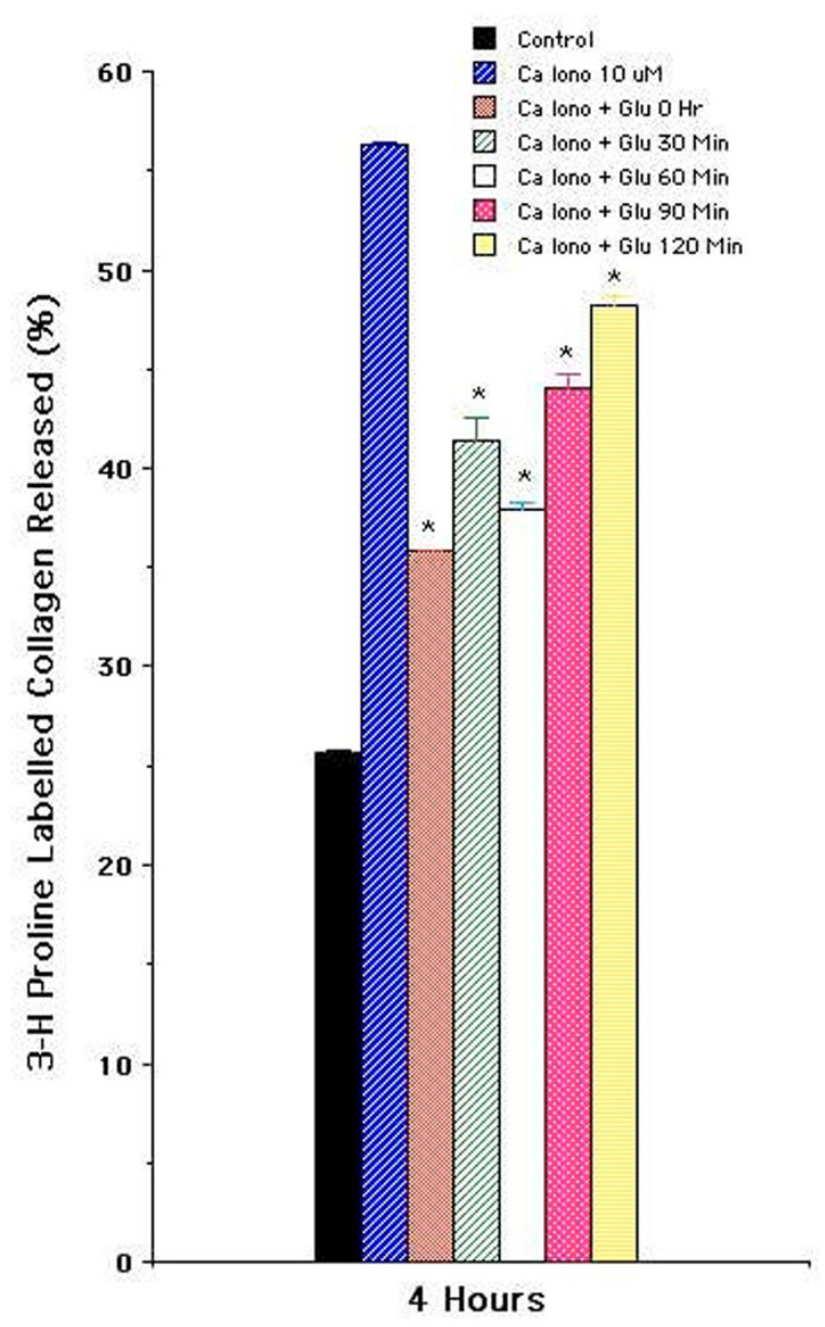

Time-dependent inhibitory effect of glucosamine hydrochloride on the release of $\left[{ }^{3} \mathrm{H}\right]$-proline-labeled articular collagen matrix. $\left[{ }^{3} \mathrm{H}\right]$-prolinelabeled monolayer of primary articular chondrocytes was stimulated with A23187 $(10 \mu \mathrm{M})$ in the absence and presence of glucosamine hydrochloride $(50 \mathrm{mM})$. Glucosamine was added at the initiation (0 hours) or at different times as shown in the figure. The 4-hour percentage release of labeled matrix (collagen) is shown. The results are presented as the mean of triplicate set of wells \pm standard error. A representative experiment is shown. *Statistically significant between cells stimulated with calcium ionophore and in the presence of glucosamine hydrochloride. Ca lono, calcium ionophore; Glu, glucosamine hydrochloride.

\section{Glucosamine hydrochloride does not inhibit conjugated diene formation by activated chondrocytes and lipoprotein oxidation}

We monitored conjugated diene formation as an indicator of lipid peroxidation in activated chondrocytes and purified lipoprotein oxidation with or without glucosamine hydrochloride [32]. As shown in Figure 4a, calcium ionophore-stimulated chondrocytes resulted in progressive increase in the conjugated diene formation. Glucosamine hydrochloride $(50 \mathrm{mM})$ did not inhibit conjugated diene formation in stimulated chondrocytes. Vitamin $E(250 \mu \mathrm{M})$ inhibited conjugated diene formation in stimulated chondrocytes. Of note, glucosamine hydrochloride had a slight stimulatory effect on conjugated diene formation as compared with the release of conjugated diene by unstimulated control chondrocytes. There was no inhibition of conjugated diene formation in Cu-induced oxidation of purified lipoproteins by glucosamine hydrochloride (Figure 4b). Together, the data indicate that glucosamine does not inhibit initiation or progression of lipid peroxidation in chondrocytes or lipoproteins.

\section{Glucosamine hydrochloride inhibits TBARS formation by copper-induced lipoprotein oxidation}

We investigated the effect of glucosamine hydrochloride on TBARS formation in Cu-induced oxidation of lipoproteins. As shown in Figure 5, there was a dose-dependent inhibition of TBARS (malondialdehyde) formation by glucosamine hydrochloride. Glucosamine hydrochloride in 5 to $50 \mathrm{mM}$ concentrations resulted in almost complete inhibition of TBARS formation, whereas glucosamine hydrochloride concentration of $0.5 \mathrm{mM}$ had no inhibitory effect. The data suggest that glucosamine hydrochloride either interferes with the formation of downstream aldehydic products of lipid peroxidation or scavenges these products. It should be noted that glucosamine hydrochloride did not interfere in the detection of control malondialdehyde from the tetramethoxypropane standard.

\section{Immunoblot analysis of the effect of glucosamine hydrochloride on aldehyde-protein adduct in chondrocyte matrix extracts}

We tested the effect of glucosamine hydrochloride on aldehyde-protein adduct formation in control and stimulated chondrocytes. Protein gel electrophoresis and immunoblot analysis using MDA2, specific for MDA-modified lysine of chondrocyte extracts, is shown in Figure 6. Extracts from control chondrocytes with glucosamine resulted in a slight increase in background immunoreactive bands to MDA2. Extracts from calcium ionophore-stimulated chondrocytes resulted in a further increase in immunoreactivity and in the appearance of new low-molecular-weight immunoreactive bands to MDA2. Increased reactivity and appearance of lowmolecular-weight aldehyde-protein adducts suggest activation-dependent aldehydic protein oxidation and protein fragmentation. In comparison, extracts from calcium ionophorestimulated chondrocyte matrix in the presence of glucosamine hydrochloride showed diminished presence and the disappearance of low-molecular-weight immunoreactive bands, suggesting that glucosamine hydrochloride diminishes aldehydic protein oxidation and fragmentation in activated chondrocyte extracts.

\section{Western blot analysis of effect of glucosamine on protein oxidation}

We tested the effect of glucosamine hydrochloride on lipoprotein protein oxidation using the identification of protein 

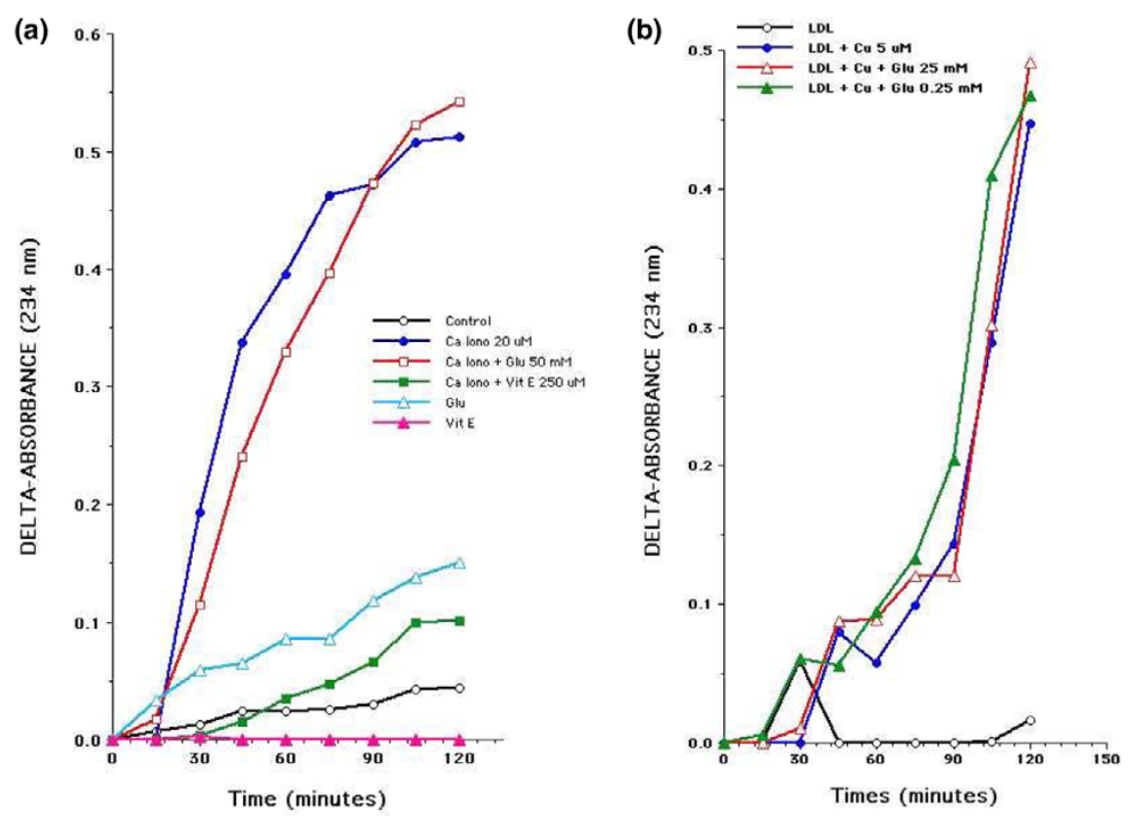

Glucosamine hydrochloride does not prevent conjugated diene formation by calcium ionophore-stimulated chondrocytes (a) or by copper-catalyzed oxidation of low-density lipoprotein (b). (a) A washed monolayer of primary articular chondrocytes in 60-mm Petri dishes was stimulated in the presence or absence of A23187 $(20 \mu \mathrm{m})$ with or without glucosamine $(50 \mathrm{mM})$ or Vitamin $E(250 \mu \mathrm{M})$ in phenol-free Earl's balanced salt solution. The media were monitored for conjugated diene formation at $234 \mathrm{~nm}$ at different time points. Delta absorbance shown is absorbance at different time points minus the absorbance at 0 hours. A representative of four experiments is shown. (b) Low-density lipoprotein $(0.25 \mathrm{mg} / \mathrm{ml})$ was incubated at $30^{\circ} \mathrm{C}$ in phosphate-buffered saline alone (open circles) or in the presence of $5 \mu \mathrm{M} \mathrm{Cu}^{2+}$ (closed circles) or with $5 \mu \mathrm{M} \mathrm{Cu}{ }^{2+}$ and $25 \mathrm{mM}$ (open triangles) or $0.25 \mathrm{mM}$ (closed triangles) glucosamine. A conjugated diene formation was monitored at $234 \mathrm{~nm}$. Ca lono, calcium ionophore; Cu, copper; Glu, glucosamine; LDL, low-density lipoprotein; Vit E, vitamin E.

carbonyls as one of the modifications as described in oxidized proteins [33,34]. The carbonyl groups generated on oxidized proteins were allowed to react with 2,4-dinitrophenylhydrazine and this group is recognized by anti-DNP antibodies [31]. As shown in the DNP immunoblot in Figure 7, the addition of glucosamine hydrochloride alone did not generate carbonyl modification in lipoproteins as compared with control. Lipoproteins oxidized with $\mathrm{Cu}$ resulted in diffused DNP immunoreactivity to high-molecular-weight lipoproteins (as indicated by the arrow in lane 3). This diffused DNP immunoreactivity was obliterated by a $50 \mathrm{mM}$ concentration of glucosamine hydrochloride in Cu-oxidized lipoproteins (as seen in lane 4), suggesting that glucosamine prevents formation of carbonyl groups in oxidized proteins. On the other hand, glucosamine hydrochloride in concentrations of $5.0 \mathrm{mM}$ or $0.5 \mathrm{mM}$ had little effect on DNP immunoreactivity (as seen in lanes 5 and 6). Two bands of lowmolecular-weight DNP immunoreactive bands were observed in control and $\mathrm{Cu}$-stimulated lipoproteins, and glucosamine had no discernable effect on their signal intensity.

\section{Discussion}

Using this in vitro model of chondrocyte activation-dependent collagen degradation, we show that glucosamine specifically and significantly inhibited collagen degradation. Inhibition of collagen degradation by glucosamine was not mediated by inhibiting the chondrocyte lipid peroxidation process but by inhibiting advanced lipoxidation reactions. Specifically, glucosamine inhibited purified lipoprotein protein oxidation and aldehydic oxidation of chondrocyte matrix.

Using this in vitro model, we had previously shown $[26,35,36]$ that chondrocyte-derived lipid radicals specifically mediate degradation of cartilage collagen [26,35]. This model therefore is a fair representation of cartilage collagen degradation. The relevance of this in vitro model to human OA pathogenesis was demonstrated by detection of in vivo molecular imprints of lipid peroxidation in which $\mathrm{OA}$ and normal cartilage tissue sections were studied [36]. We also demonstrated the presence of OA disease-specific malondialdehyde and hydroxynonenal adducts in human OA cartilage tissue sections, suggesting the in vivo role of lipid peroxidation in the OA pathogenesis $[36,37]$. Collectively, these observations indicate that lipid peroxidation may play a larger role in the pathogenesis OA than has previously been recognized.

We investigated the effect of glucosamine in our assay system. As shown, only glucosamine hydrochloride or glucosamine sulfate specifically and significantly inhibited collagen degradation by activated chondrocytes and the effect was dose-dependent. Similar effects by both agents (glucosamine hydrochloride and glucosamine sulfate) excluded the possibility that the inhibition observed was medi- 
Figure 5

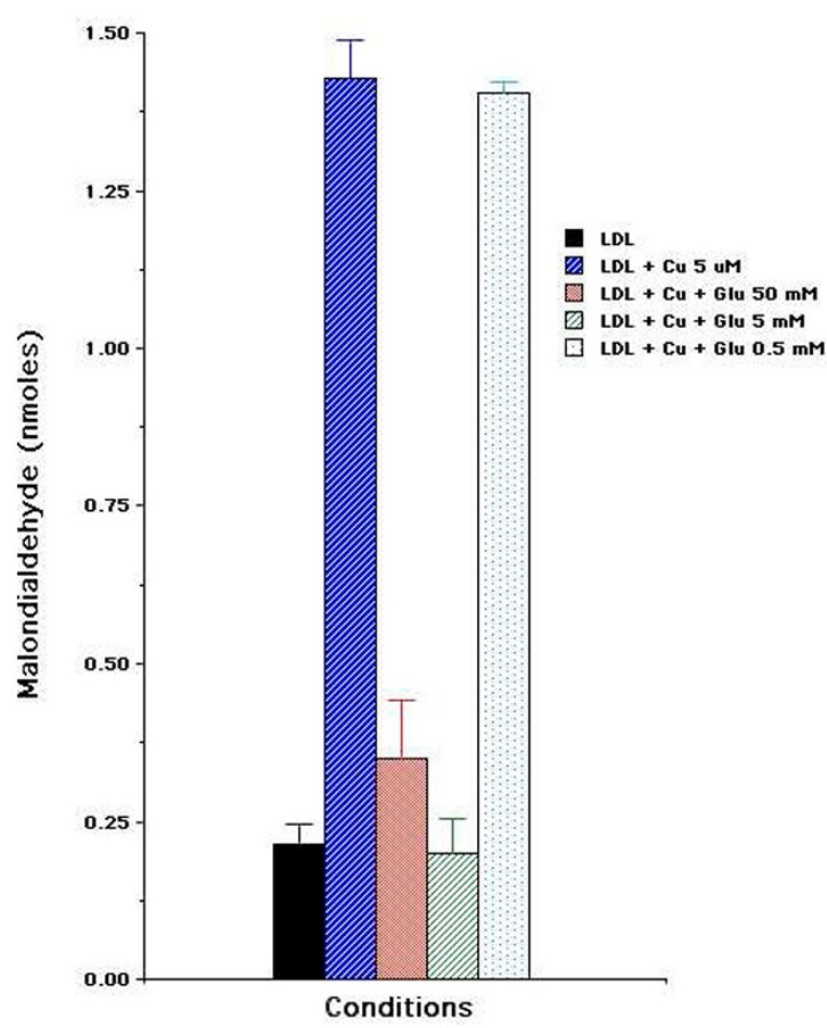

Glucosamine hydrochloride inhibits malondialdehyde formation by lipoprotein oxidation. Lipoproteins $(0.5 \mathrm{mg} / \mathrm{ml})$ were incubated at $30^{\circ} \mathrm{C}$ in phosphate-buffered saline for 4 hours in the absence or presence of 5 $\mu \mathrm{M} \mathrm{Cu}^{2+}$ or $5 \mu \mathrm{M} \mathrm{Cu}^{2+}$ and 50,5 , or $0.5 \mathrm{mM}$ glucosamine hydrochloride. Data are expressed as malondialdehyde equivalents in nanomoles and are presented as the mean of a duplicate set of samples \pm standard error. A representative of two experiments is shown. $\mathrm{Cu}$, copper; Glu, glucosamine hydrochloride; LDL, low-density lipoprotein.

ated by the sulfate moiety in the latter compound. Glucosamine hydrochloride had little or variable effect on hydrogen peroxide-induced collagen degradation, suggesting that it did not inhibit oxygen radical/hydrogen peroxide-mediated collagen degradation (data not shown).

Since the mechanism of collagen degradation in this model appears to involve the activation of lipid peroxidation in chondrocytes, it raises the possibility that glucosamine was acting like a chain-breaking antioxidant similar to vitamin $E$. However, glucosamine had no discernable effect on conjugated diene formation by activated chondrocytes, suggesting that its mechanism of action was not due to chain-breaking antioxidant activity. As expected, vitamin $E$ inhibited conjugated diene formation by chondrocytes. To further confirm these findings, we tested the effect of glucosamine in a purified lipoprotein oxidation model system, a commonly used in vitro model for studies on lipoxidative modification of proteins [27]. Again, glucosamine hydrochloride had no discernable effect on $\mathrm{Cu}$-induced conjugated diene formation in lipopro-
Figure 6

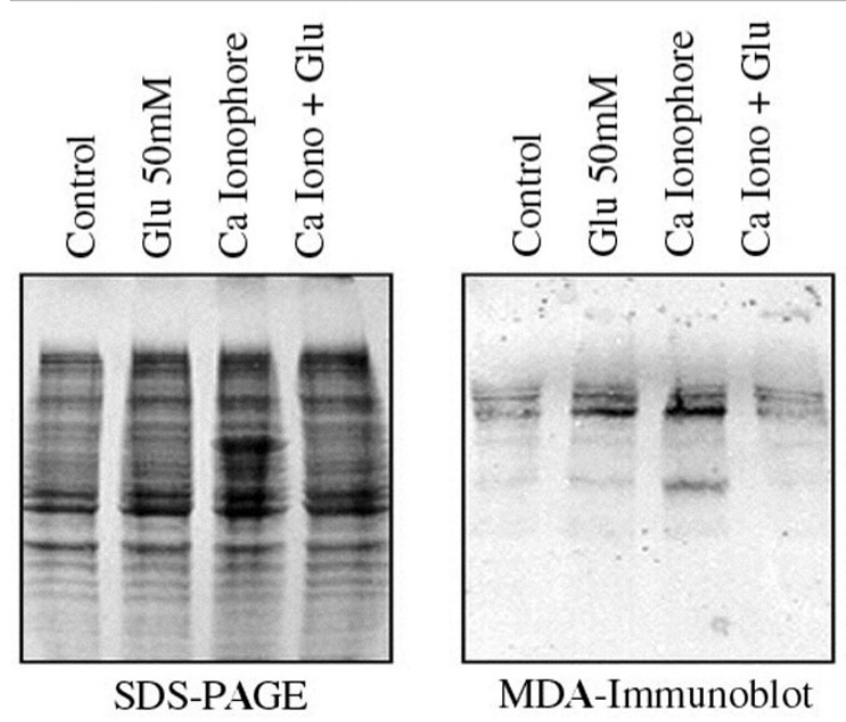

SDS-PAGE and subsequent immunoblot analysis of chondrocyte extracts. Primary confluent articular chondrocytes in 60-mm Petri dishes were washed and finally set in serum-free Earl's balanced salt solution without (control, lane 1) or with glucosamine hydrochloride (50 $\mathrm{mM}$, lane 2) or with $\mathrm{Ca}$ lono $(20 \mu \mathrm{M}$, lane 3$)$ and $\mathrm{Ca}$ lono with glucosamine hydrochloride (lane 4). The chondrocytes were stimulated for 4 hours. Extracts of media-cell matrix were collected as described, and $30 \mu \mathrm{l}$ of extracts was loaded on SDS-PAGE and transblotted onto nitrocellulose membrane. Subsequently, the membranes were reacted with MDA2 monoclonal antibodies overnight and were processed. $\mathrm{Ca}$ Iono, calcium ionophore; Glu, glucosamine hydrochloride.

teins. Furthermore, glucosamine did not cause an increase in the lag phase of LDL oxidation or a decrease in absorbance at $234 \mathrm{~nm}$ during the later plateau phase of the reaction. Together, these observations indicate that glucosamine does not interfere with initiation or propagation of lipid peroxidation reaction.

The inhibition of collagen degradation by glucosamine was manifested even when the addition of glucosamine was delayed in activated chondrocyte cultures, indicating that its mechanism of action involved downstream events of chondrocyte activation rather than interfering with or blocking the early events of chondrocyte activation by calcium ionophore. We tested the effect of glucosamine on TBARS formation by $\mathrm{Cu}$ induced oxidation of purified lipoproteins. Glucosamine in a dose-dependent manner inhibited malondialdehyde formation by oxidized lipoprotein. The data suggest that glucosamine either inhibited or scavenged aldehydic products of lipid peroxidation. However, glucosamine did not interfere in the detection of control malondialdehyde in TBARS assay, suggesting that most likely glucosamine inhibited advanced lipoxidation reactions rather than scavenging aldehydic products.

The identification of aldehydic adducts provides a molecular clue of chondrocyte matrix damage mediated by lipid-free rad- 


\section{Figure 7}

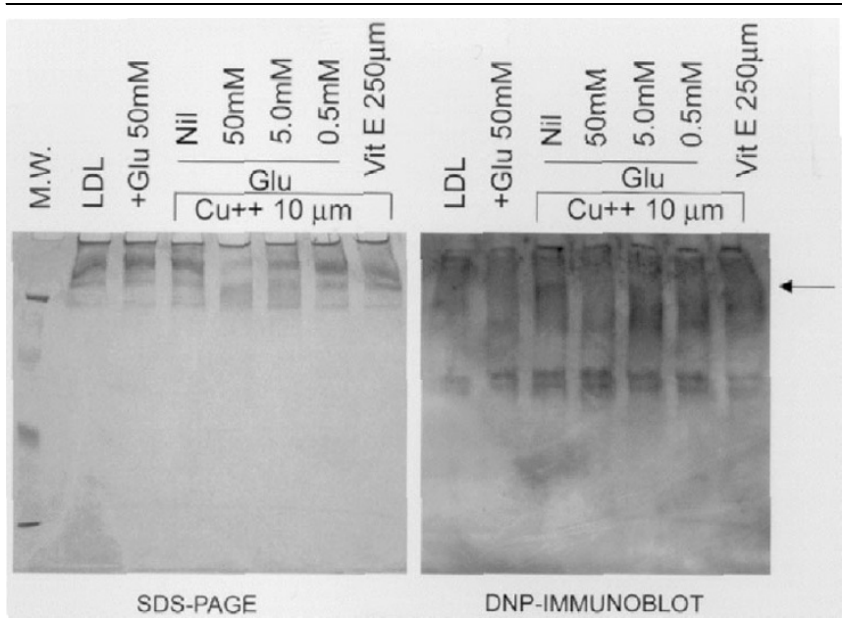

SDS-PAGE and subsequent immunoblot analysis of lipoproteins. Lipoproteins $(200 \mu \mathrm{g})$ in a total volume of $200 \mu \mathrm{l}$ were incubated with or without calcium $(10 \mu \mathrm{M})$ in the absence or presence of variable concentrations of glucosamine hydrochloride for 4 hours at $30^{\circ} \mathrm{C}$. The reaction was stopped by the addition of EDTA (ethylenediaminetetraacetic acid) with butylated hydroxytoluene, and aliquots were stored at $-70^{\circ} \mathrm{C}$. Thawed samples were derivatized with DNP, and $40 \mu$ of sample was loaded on SDS-PAGE and transblotted onto nitrocellulose membranes. Subsequently, the membrane was incubated with anti-DNP antibodies for 1 hour and processed. DNP, dinitrophenyl; Glu, glucosamine hydrochloride; LDL, low-density lipoprotein; M.W., molecular weight; Vit E, vitamin E. Arrow indicates diffused DNP reactivity to high molecular weight lipoproteins in Lane 3.

icals [26]. On immunoblot analysis of the effect of glucosamine, we identified activation-dependent low-molecularweight MDA adduct in chondrocyte matrix extracts; the intensity of higher-molecular-weight aldehydic adducts increased in activated chondrocyte extracts as compared with extracts from control chondrocyte matrix. In the presence of glucosamine, the low-molecular-weight aldehydic adducts in activated extracts disappeared whereas the intensity of highmolecular-weight adducts decreased, indicating that glucosamine prevented oxidation and/or fragmentation of chondrocyte matrix components. These observations are consistent with the finding that glucosamine inhibited malondialdehyde (TBARS) formation in Cu-induced oxidation of lipoprotein. Together, these observations suggest that glucosamine inhibits advanced lipoxidation reactions. By preventing advanced lipid-free radical production, glucosamine perhaps inhibits collagen degradation observed in the in vitro model system.

Inhibitors of advanced lipoxidation reactions such as aminoguanidine and pyridoxamine have been evaluated in animal models of diseases such as diabetes $[38,39]$. These compounds are being evaluated in clinical trials for the treatment of diabetic nephropathy [40]. Aminoguanidine inhibits chemical modification of proteins during lipid peroxidation reactions and inhibits metal-catalyzed oxidation of LDLs and uptake of oxidized LDL into macrophages via the scavenger receptor
[41,42]. Pyridoxamine has also been shown to have potent advanced lipoxidation inhibitory activities in a variety of tests $[38,39]$. In addition to showing advanced lipoxidation inhibitory activity, these compounds show inhibitory activity against advanced glycation reactions (AGEs) $[38,43]$. AGE products formed during autoxidation of carbohydrates and lipid peroxidation reactions produce reactive carbonyl species that cause a carbonyl modification reaction in protein structure and function and cause the formation of high-molecular-weight protein aggregates [33]. Osteoarthritic cartilage shows increased levels of insoluble protein aggregates and AGE-modified products [44-46]. Identification of carbonyl modification of proteins provides a powerful tool to monitor the development of a number of pathologies mediated by a condition commonly described as 'carbonyl stress' [33,34,47]. As shown, glucosamine inhibited Cu-induced carbonyl modification of lipoproteins, indicating that glucosamine also traps reactive carbonyl compounds. In addition to aminoguanidine and pyridoxamine, therapeutic agents such as L-arginine, OPB-9195, tenilsetam, and metformin have been proposed to trap reactive carbonyl compounds [48-53].

The pharmacokinetics of oral administration of glucosamine sulfate show that plasma levels increase more than 30 -fold from baseline and peak at approximately $10 \mu \mathrm{M}$ with the standard 1,500-mg once-daily dosage [54]. We postulate that because in vivo tissue levels of glycosaminoglycans in cartilage are hundreds perhaps thousands of folds higher than in serum or joint fluids, glucosamine, which is a structural component of aggrecan, may locally provide an antioxidant environment that may protect cartilage collagen from oxidative damage.

Our data suggest that the decrease in collagen degradation by glucosamine observed in this in vitro model system may be mediated by the inhibition of advanced lipoxidation reaction, preventing the oxidation and loss of collagen matrix from labeled chondrocyte matrix. Further studies are needed to relate these in vitro findings to the retardation of cartilage degradation reported in OA trials investigating glucosamine.

\section{Conclusion}

In an in vitro model of cartilage collagen degradation in which collagen degradation induced by activated chondrocytes is mediated by lipid peroxidation reaction, glucosamine decreases collagen degradation by inhibiting advanced lipoxidation reaction and thus prevents the oxidation and loss of collagen matrix from labeled chondrocyte matrix.

\section{Competing interests}

The authors declare that they have no competing interests.

\section{Authors' contributions}

MLT developed the study experimental protocol. All authors participated in conducting and analyzing the experiments. All 
authors were involved in the drafting, review, and final approval of the manuscript.

\section{References}

1. Hamerman D: The biology of osteoarthritis. N Engl J Med 1989, 320:1322-1330.

2. Centers for Disease Control and Prevention (CDC): Update: direct and indirect costs of arthritis and other rheumatic conditions - United States, 1997. MMWR Morb Mortal Wkly Rep 2004, 53:388-389.

3. Reginster JY, Deroisy R, Rovati LC, Lee RL, Lejeune E, Bruyere O, Giacovelli G, Henrotin Y, Dacre JE, Gossett C: Long-term effect of glucosamine sulfate on osteoarthritis progression: a randomised, placebo-controlled clinical trial. Lancet 2001, 357:251-256.

4. Pavelká K, Gatterová J, Olejarová M, Machacek S, Giacovelli G, Rovati LC: Glucosamine sulfate use and delay of progression of knee osteoarthritis: a 3-year, randomized, placebo-controlled, double-blind study. Arch Intern Med 2002, 162:2113-2123.

5. Bruyere O, Pavelka K, Rovati LC, Deroisy R, Olejarova M, Gatterova J, Giacovelli G, Reginster JY: Gluocosamine sulfate reduces osteoarthitis progression in postmenopausal women with knee osteoarthritis: evidence from two 3-year studies. Menopause 2004, 11:138-143.

6. Altman RD, Abramson S, Bruyere O, Clegg D, Herrero-Beaumont G, Maheu E, Moskowitz R, Pavelka K, Reginster JY: Commentary: osteoarthritis of the knee and glucosamine. Osteoarthritis Cartilage 2006, 14:963-966.

7. Clegg DO, Reda DJ, Harris CL, Klein MA, O'Dell JR, Hooper MM, Bradley JD, Bingham CO 3rd, Weisman MH, Jackson CG, et al.: Glucosamine, chondroitin sulfate, and two in combination for painful knee osteoarthritis. N Engl J Med 2006, 354:795-808.

8. Herrero-Beaumont G, Ivorra JA, Del Carmen Trabado M, Blanco FJ, Benito P, Martín-Mola E, Paulino J, Marenco JL, Porto A, Laffon $A$, et al:: Glucosamine sulfate in the treatment of knee osteoarthritis symptoms: a randomized, double-blind, placebo-controlled study using acetaminophen as a side comparator. Arthritis Rheum 2007, 56:555-567.

9. Shikhman AR, Kuhn K, Alaaeddine N, Lotz M: N-acetylglucosamine prevents IL-1 beta-mediated activation of human chondrocytes. J Immunol 2001, 166:5155-5160.

10. Meininger CJ, Kelly KA, Li H, Haynes TE, Wu G: Glucosamine inhibit inducible nitric oxide synthesis. Biochem Biophys Res Commun 2000, 279:234-239.

11. Piperno M, Reboul P, Hellio Le Graverand MP, Peschard MJ, Annefeld M, Richard M, Vignon E: Glucosamine sulfate modulates dysregulated activities of human osteoarthritic chondrocytes in vitro. Osteoarthritis Cartilage 2000, 8:207-212.

12. Ilic MZ, Martinac B, Handley CJ: Effect of long-term exposure to glucosamine and mannosamine on aggrecan degradation in articular cartilage. Osteoarthritis Cartilage 2003, 11:613-622.

13. Fenton JI, Chlebek-Brown KA, Peters TL, Caron JP, Orth MW: Glucosamine $\mathrm{HCL}$ reduces equine articular cartilage degradation in explant culture. Osteoarthritis Cartilage 2000, 8:258-265.

14. Bassleer C, Rovati L, Franchimont P: Stimulation of proteoglycan production by glucosamine sulfate in chondrocytes isolated from human osteoarthritic articular cartilage in vitro. Osteoarthritis Cartilage 1998, 6:427-434.

15. Dodge GR, Jimenez SA: Glucosamine sulfate modulates the levels of aggrecan and matrix metalloproteinase-3 synthesized by cultured human osteoarthritis articular chondrocytes. Osteoarthritis Cartilage 2003, 11:424-432.

16. Sandy JD, Gamett D, Thompson V, Verscharen C: Chondrocytemediated catabolism of aggrecan: aggrecanase-dependent cleavage induced by interleukin-1 or retinoic acid can be inhibited by glucosamine. Biochem J 1988, 335:59-66.

17. Sandy JD, Thompson V, Verscharen C, Gamett D: Chondrocytemediated catabolism of aggrecan: evidence for a glycosylphosphatidylinositol-linked protein in the aggrecanase response to interleukin-1 or retinoic acid. Arch Biochem Biophys 1999, 367:258-264.

18. Gouze JN, Bianchi A, Bécuwe P, Dauça M, Netter P, Magdalou J, Terlain B, Bordji K: Glucosamine modulates IL-1 induced activation of rat chondrocytes at receptor level, and by inhibiting the NF-kappa B pathway. FEBS Lett 2002, 510:166-170.
19. Largo R, Alvarez-Soria MA, Diez-Ortego I, Calvo E, Sánchez-Pernaute $O$, Egido J, Herrero-Beaumont G: Glucosamine inhibits IL1 beta-induced NFkappaB activation in human osteoarthritic chondrocytes. Osteoarthritis Cartilage 2003, 11:290-298.

20. Lippiello L, Woodward J, Karpman R, Hammad TA: In vivo chondroprotection and metabolic synergy of glucosamine and chondroitin sulfate. Clin Orthop Relat Res 2000, 381:229-240.

21. Shikhman AR, Amiel D, D'Lima D, Hwang SB, Hu C, Xu A, Hashimoto S, Kobayashi K, Sasho T, Lotz MK: Chondroprotective activity if $\mathrm{N}$-acetylglucosamine in rabbits with experimental osteoarthritis. Ann Rheum Dis 2005, 64:89-94.

22. Tamai $Y$, Miyatake K, Okamoto $Y$, Takamori $Y$, Sakamoto H, Minami $S$ : Enhanced healing of cartilaginous injuries by glucosamine hydrochloride. Carbohydrate Polymers 2002, 48:369-378.

23. Tiraloche G, Girard C, Chouinard L, Sampalis J, Moquin L, lonescu M, Reiner A, Poole AR, Laverty S: Effect of oral glucosamine on cartilage degradation in rabbit model of osteoarthritis. Arthritis Rheum 2005, 52:1118-1128.

24. Ma L, Rudert WA, Harnaha J, Wright M, Machen J, Lakomy R, Qian $\mathrm{S}$, Lu L, Robbins PD, Trucco M, et al:: Immunosuppressive effects of glucosamine. J Biol Chem 2002, 277:39343-39349.

25. Quastel JH, Cantero A: Inhibition of tumour growth by D-glucosamine. Nature 1953, 171:252-254.

26. Tiku ML, Shah R, Allison GT: Evidence linking chondrocyte lipid peroxidation to cartilage matrix protein degradation. Possible role in cartilage aging and the pathogenesis of osteoarthritis. $J$ Biol Chem 2000, 275:20069-20076.

27. Rifici VA, Khachadurian AK: Dietary supplementation with vita$\min \mathrm{C}$ and $\mathrm{E}$ inhibit in vitro oxidation of lipoproteins. J Am Coll Nutr 1993, 12:631-637.

28. Lavy A, Brook GJ, Dankner G, Ben Amotz A, Aviram M: Enhanced in vitro oxidation of plasma lipoproteins derived from hypercholesterolemic patients. Metabolism 1991, 40:794-799.

29. Laemmli UK: Cleavage of structural proteins during the assembly of head of bacteriophage T4. Nature 1970, 227:680-685.

30. Palinski W, Hörkkö S, Miller E, Steinbrecher UP, Powell HC, Curtiss LK, Witztum JL: Cloning of monoclonal autoantibodies to epitopes of oxidized lipoproteins from apolipoprotein E-deficient mice. Demonstration of epitopes of oxidized low density lipoprotein in human plasma. J Clin Invest 1996, 98:800-814.

31. Shacter EY, Williams JA, Stadtman ER: Determination of carbonyl groups in oxidized proteins. In Free Radicals: A Practical Approach Edited by: Punchard NA, Kelly FJ. Oxford University Press, New York: IRL Press; 1996:159-170.

32. Gutteridge JM: Lipid peroxidation and antioxidants as biomarkers of tissue damage. Clin Chem 1995, 41:1819-1828.

33. Berlett BS, Stadtman ER: Protein oxidation in aging, disease, and oxidative stress. J Biol Chem 1997, 272:20313-20316.

34. Tamarit J, Cabiscol E, Ros J: Identification of major oxidatively damaged proteins in Escherichia coli cells exposed to oxidative stress. J Biol Chem 1998, 273:3027-3032.

35. Tiku ML, Allison GT, Naik K, Karry SK: Malondialdehyde oxidation of cartilage collagen by chondrocytes. Osteoarthritis Cartilage 2003, 11:159-166.

36. Shah R, Raska K Jr, Tiku ML: The presence of molecular markers of in vivo lipid peroxidation in osteoarthritic cartilage: a pathogenic role in osteoarthritis. Arthritis Rheum 2005, 52:2799-2807.

37. Morquette $B$, Shi $Q$, Lavigne $P$, Ranger $P$, Fernandes JC, Benderdour M: Production of lipid peroxidation products in osteoarthritic tissues: new evidence linking 4-hydroxynonenal to cartilage degradation. Arthritis Rheum 2006, 54:271-281.

38. Onorato JM, Jenkins AJ, Thorpe SR, Baynes JW: Pyridoxamine, an inhibitor of advanced glycation reactions, also inhibits advanced lipoxidation reactions. Mechanism of action of pyridoxamine. J Biol Chem 2000, 275:21177-21184.

39. Metz TO, Alderson NL, Chachich ME, Thorpe SR, Baynes JW: Pyridoxamine traps intermediates in lipid peroxidation reactions in vivo: evidence on the role of lipids in chemical modification of protein and development of diabetic complications. $J$ Biol Chem 2003, 278:42012-42019.

40. Degenhardt TP, Alderson NL, Arrington DD, Beattie RJ, Basgen JM, Steffes MW, Thorpe SR, Baynes JW: Pyridoxamine inhibits early renal disease and dyslipidemia in streptozotocin-diabetic rat. Kidney Int 2002, 61:939-950.

41. Requena JR, Vidal P, Cabezas-Cerrato J: Aminoguanidine inhibits the modification of proteins by lipid peroxidation derived 
aldehyde: a possible anti-atherogenic agent. Diabetes Res 1992, 20:43-49.

42. Picard S, Parthasarathy S, Fruebis J, Witztum JL: Aminoguanidine inhibits oxidative modification of low-density lipoprotein protein and the subsequent increase in uptake by macrophage scavenger receptors. Proc Natl Acad Sci USA 1992, 89:6876-6880.

43. Voziyan PA, Metz TO, Baynes JW, Hudson BG: A post-Amadori inhibitor pyridoxamine also inhibits chemical modification of proteins by scavenging carbonyl intermediated of carbohydrates and lipid degradation. J Biol Chem 2002, 277:3397-3403.

44. Uchiyama A, Ohishi T, Takahashi M, Kushida K, Inoue T, Fujie M, Horiuchi K: Fluorophores from aging human cartilage. J Biochem (Tokyo) 1991, 110:714-718.

45. Hormel SE, Eyre DR: Collagen in the ageing human intervertebral disc: an increase in covalently bound fluorophores and chromophores. Biochim Biophys Acta 1991, 1078:243-250.

46. Pokharna HK, Monnier V, Boja B, Moskowitz RW: Lysyl oxidase and Maillard reaction-mediated crosslinks in aging and osteoarthritic rabbit cartilage. J Orthop Res 1995, 13:13-21.

47. Baynes JW, Thorpe SR: Role of oxidative stress in diabetic complications: a new perspective on an old paradigm. Diabetes 1999, 48:1-9.

48. Nakamura S, Makita Z, Ishikawa S, Yasumura K, Fujii W, Yanagisawa K, Kawata T, Koike T: Progression of nephropathy in spontaneous diabetic rats is prevented by OPB- a novel inhibitor of advanced glycation. Diabetes 1995, 46:895-899.

49. Shoda H, Miyata S, Liu BF, Yamada H, Ohara T, Suzuki K, Oimomi $M$, Kasuga M: Inhibitory effects of tenilsetam on the Maillard reaction. Endocrinology 1997, 138:1886-1892.

50. Brownlee $M$, Vlassara $H$, Kooney A, Ulrich P, Cerami A: Aminoguanidine prevents diabetes-induced arterial wall protein cross-linking. Science 1986, 232:1629-1632.

51. Lo TW, Selwood T, Thornalley PJ: The reaction of methylglyoxal with aminoguanidine under physiological conditions and prevention of methylglyoxal binding to plasma proteins. Biochem Pharmacol 1994, 48:1865-1870.

52. Lubec B, Aufricht $C$, Amann G, Kitzmüller E, Höger H: Arginine reduces kidney collagen accumulation, cross-linking, lipid peroxidation, glycoxidation, kidney weight and albuminuria in diabetic KK mouse. Nephron 1997, 75:213-218.

53. Ruggiero-Lopez D, Lecomte M, Moinet G, Patereau G, Lagarde M, Wiernsperger N: Reaction of metformin with dicaronyl compounds. Possible implication in the inhibition of advanced glycation end product formation. Biochem Pharmacol 1999, 58:1765-1773.

54. Persiani S, Roda E, Rovati LC, Locatelli M, Giacovelli G, Roda A: Glucosamine oral bioavailability and plasma pharmacokinetics after increasing doses of crystalline glucosamine sulfate in man. Osteoarthritis Cartilage 2005, 13:1041-1049. 